\title{
Electronic Tongue and Neural Networks, Biologically Inspired Systems Applied to Classifying Coffee Samples
}

\author{
Álvaro A. Arrieta Almario ${ }^{*}$, Rosa L. Tarazona Cáceres ${ }^{2}$ \\ ${ }^{1}$ Grupo de Investigación DANM/Desarrollo y Aplicación de Nuevos Materiales, Universidad Pontificia \\ Bolivariana, Montería, Colombia \\ ${ }^{2}$ Grupo de Investigación GIE, Universidad Pontificia Bolivariana, Montería, Córdoba \\ Email: ${ }^{*}$ alvaroangel.arrieta@gmail.com
}

Received 9 February 2014; revised 10 March 2014; accepted 17 March 2014

Copyright (C) 2014 by authors and Scientific Research Publishing Inc.

This work is licensed under the Creative Commons Attribution International License (CC BY). http://creativecommons.org/licenses/by/4.0/

(c) (i) Open Access

\begin{abstract}
In this paper, the possibility to use an electronic tongue based on a polypyrrole sensor array in classifying coffee samples has been studied. Each sensor shows a distinguished electrochemical response when exposed to the studied solutions, providing signals with a high degree of cross-selectivity. The sensor array electrochemical response is related to the interaction of the ionic and non-ionic solution compounds and to the surface of the sensors polymeric matrix. Furthermore, the electronic tongue was used to perform an analysis on coffee samples. In this case, each sensor showed a particular response to each coffee sample. Data obtained from the registered signals were used to perform a discrimination of the samples. The analysis with neural networks of the principal components (NNPC) done on the electronic tongue response to five types of commercial coffee, allows to achieve a clear differentiation of samples.
\end{abstract}

\section{Keywords}

Electronic Tongue; Coffee; Electrochemical Sensors

\section{Introduction}

In the food industry, the commercial success of a product is determined by many factors, such as appearance, aroma and flavor. The growing customers' demand on food quality makes the inspection and approval of products more rigorous every day. In order to guarantee the quality of commercial drinks, specifically coffee, differ-

${ }^{*}$ Corresponding author.

How to cite this paper: Almario, Á.A.A. and Cáceres, R.L.T. (2014) Electronic Tongue and Neural Networks, Biologically Inspired Systems Applied to Classifying Coffee Samples. American Journal of Analytical Chemistry, 5, 266-274. 
ent methods of chemical, Physicochemical and sensory analysis are utilized. The first ones include a series of well-known techniques, mainly volumetric, chromatographic and spectroscopic, which are able to categorize with great accuracy the chemical composition. The goal of the second ones—sensory methods-is to evaluate the sensations produced by coffee altogether [1]. The sensorial analysis can be defined as the experimentation and analysis of the global characteristics of a product through the senses; those characteristics are known as organoleptic.

Smell and taste senses are known as chemical senses since they respond in a direct manner to stimuli produced by molecules originating a nerve reaction. Taste responds to stimuli produced by food molecules on the tongue's taste cells. That interaction produces the sensation of taste. The taste receptor organs are located above all on the taste buds. The mechanism by which different flavors are detected and recognized is not yet determined, but it is known that taste cells respond with partial selectivity to a series of chemical compounds; in detecting a flavor a series of electrical impulses are generated with different intensities and are transmitted to the brain, where they are compared to identify flavors [2] [3].

Smell and taste systems have produced inspiration so that from its functioning principles instruments may be developed to search for a way to emulate its capacity to classify and differentiate complex substances [4]-[6]. These biologically inspired systems are known as electronic noses and tongues. Thus, a certain analogy can be established between the human taste system and the electronic one in order to detect flavors (electronic tongues), so that some approximations can be found in its structure and functioning principles (Figure 1).

Electronic systems to measure flavors or electronic tongues are some of the most promising tendencies to develop a fast, cheap and objective method to evaluate food taste. Since the development of the first prototype in 1990 by K. Toko, [7] a certain number of research groups have focused their efforts on the improvement andperfection of those systems by utilizing diverse strategies and measuring techniques. There are several types of sensors that can be utilized in electronic tongues. The most important ones are based on electrochemical techniques such as potentiometry and voltammetry. In the bibliography there are diverse papers where these types of instruments have found numerous applications [4]-[6] [8]-[11]. However, it is also possible to use sensors based on other types of transduction, such as, optical or gravimetric sensors [12]-[14].

The information given by electronic tongue instruments is analyzed through multi-variant statistical techniques [15] [16]. The selection of the statistical analysis method depends on the type of study being carried out and the type of given data. The most utilized techniques to treat data from electronic tongues are different methods of recognizing patterns, such as the Principal Component Analysis (PCA), Cluster Analysis, Discriminant Analysis (DA) and so on. Undoubtedly, PCA is the most utilized chemometric method in studies made with electronic tongues because of its accessibility, simplicity in interpretation and low informatics cost. The Principal Component Analysis is a lineal method used to reduce variables or dimensions. It has been widely used in unnumbered applications. Nonetheless, being a lineal method, it is not always the most appropriate one because it generates redundancy when expressing data and it is possible to ignore important information when considering the array response as a lineal system. To solve this problem, some non-lineal PCA methods are proposed. However, many of the non-lineal methods have a constraint. The number of components to utilize should be pre-determined and an explicit order of the components is not given. Bearing in mind that the PCA should not be utilized in non-lineal complex systems, this project proposes the use of a non-lineal adaptive system based on neural networks.

On the other hand, a new voltammetric sensor array based on polypyrrole is utilized in this work, whose signals are rich in information because its sensors' interactions with the analyzed samples can modify the redox potential of the electrode material (polypyrrole). The polypyrrole catalytic electroactivity can ease the oxidation or reduction of compounds present in the sample, and the sensors' response is related to the capacity to generate counterions diffusion between the matrix and the analyzed sample to keep its electroneutrality. Consequently, the given information reflects the specificities of each sample and its interaction with the electrode material, providing signals that are converted in a fingerprint of each sample that allos discrimination and classification of the samples under study. Experiments were made in this research utilizing coffee samples to test the capacity to differentiate the sensor array, and neural networks are utilized as principal components for the data treatment, thus, two biologically inspired technologies are implemented to classify coffee samples.

\section{Experimental}

\subsection{Reagents and Samples}

All reagents used had a sigma-aldrich analytical quality (pyrrole, phosphotungstic acid, potassium hexacyano- 


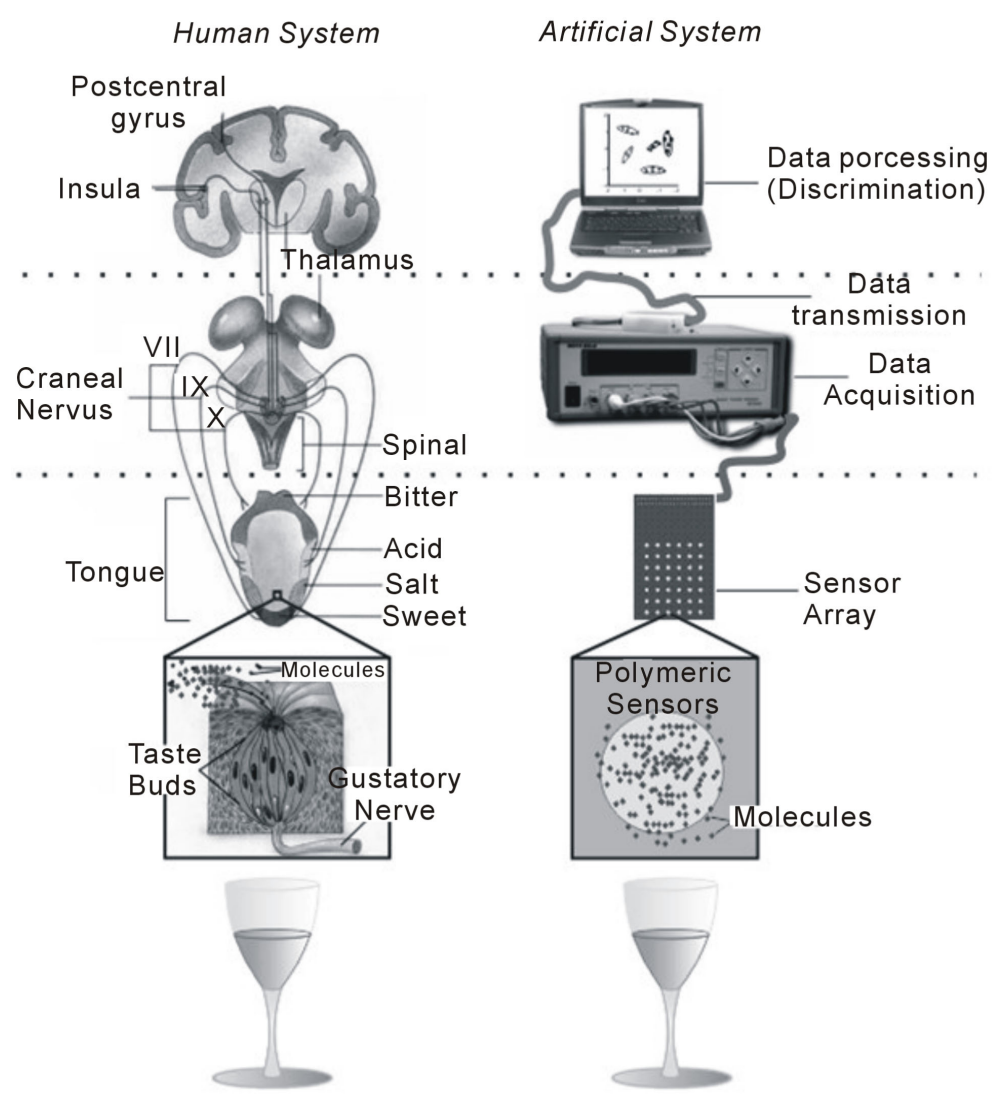

Figure 1. Functional analogy between the human taste system and the electronic system of flavors detection.

ferrate (II), sodium salt of anthraquinone-1,5-disulfonic acid, potassium ferrocyanide (III), Sodium salt of dodecylbenzensulfonic acid and sodium salt of p-toluensulfonate) and solutions were prepared with ultra purified water with a Milli-Q quality. Coffee samples were taken from five commercial brands (La Bastilla, Córdoba, Almendra Tropical, Sello Rojo and Universal) and they were prepared according to regulation NTC 3566 (sample preparation to use in sensory analysis), 7 grams of sample were weighed and prepared in $100 \mathrm{ml}$ of water. For that purpose, the water was heated in the heater up to the boiling point, $100 \mathrm{ml}$ were measured and they were poured in a beaker. The infusion was decanted for $5 \mathrm{~min}$, then remains were eliminated from the solution surface and the solution was cooled to room temperature.

\subsection{Electronic Tongue (Measuring Instrument)}

Measures on coffee samples were made with an electronic device developed in the laboratory composed by a sensor array coupled to a multichannel measuring module elaborated at LabVIEW with cards or modules for data input and output. For the acquisition of data, modules NI9263, analogous output voltage module of 16 bit and four channels, and the NI9203, input module of analogous current of 16 bit of 8 channels were used. These modules were mounted in a framework NI Compact DAQ USB especially designed for this type of cards.

The sensor array was elaborated from pyrrole electropolymerization with different doping agents. All sensors were generated by chronoamperometry in a pyrrole aqueous solution $\left(0.2 \mathrm{~mol} \mathrm{~L}^{-1}\right)$ and different doping agents were utilized in each of the sensors (each at $0.1 \mathrm{~mol} \mathrm{~L}^{-1}$ ): Phosphotungstic acid, potassium hexacyanidoferrate (II), sodium salt of anthraquinone-1,5-disulfonic acid, potassium ferrocyanide (III), Sodium salt of dodecilbencensulfonic acid and sodium salt of p-toluensulfonate. For the electropolymerization, a conventional three-electrode cell was used; reference electrode $\mathrm{Ag} / \mathrm{AgCl}$ (saturated); a platinum sheet as auxiliary electrode and as substrate (work electrode) for the polymerization, disk electrodes were used elaborated in laboratory. Polymerization conditions are summarized on Table 1. 
Table 1. Polymerization conditions of polypyrrole sensor array.

\begin{tabular}{ccccc}
\hline Sensors & Acronym & Concentration Py/Dopant (mol L $\mathbf{~}^{-\mathbf{1}}$ ) & $\mathbf{E}_{\text {polymeri.(V) }}$ & $\mathbf{t}_{\text {polymeri. }}(\mathbf{s})$ \\
\hline Pyrrole/Phosphotungstic acid & ${\mathrm{PPy} / \mathrm{SO}_{4}}$ & $0.2 / 0.05$ & $0.8 \mathrm{~V}$ & 50 \\
Pyrrole/potassium hexacyanidoferrate & PPy/HCF & $0.2 / 0.1$ & $0.8 \mathrm{~V}$ & 25 \\
Pyrrole/sodium salt of anthraquinone-1,5 disulfonic acid & PPy/AQDS & $0.2 / 0.1$ & $0.8 \mathrm{~V}$ & 50 \\
Pyrrole/potassium ferrocyanide & PPy/FCN & $0.2 / 0.1$ & $0.8 \mathrm{~V}$ & 20 \\
Pyrrole/Sodium salt of dodecilbencensulfonic acid & PPy/DBS & $0.2 / 0.1$ & $0.8 \mathrm{~V}$ & 50 \\
Pyrrole/sodium salt of p-toluensulfonate & PPy/PTS & $0.2 / 0.1$ & $0.8 \mathrm{~V}$ & 50 \\
\hline
\end{tabular}

\subsection{Measures and Data Treatment}

Coffee samples were randomly measured with the previously described instrument. After preparing samples, measures were made at room temperature, in a potential range from $-1.0 \mathrm{~V}$ to $0.5 \mathrm{~V}$ with a sweep rate of $0.1 \mathrm{~V}$ S-1. It was necessary for each measure to primarily make 10 voltamperograms to stabilize signals, and then measures were collected to feed the system of data treatment. Seven measures were made to each sample to test the method repetitiveness. In data treatment, an algorithm for neural networks was utilized for principal components available in the Statistica V7 software. Data were analyzed with no previous treatment of signals. For data interpretation, results of first components (scores) were graphed.

\section{Results and Discussion}

As established in previous works with this type of research, before starting the research on differentiation of complex substances such as coffee, which is the case in this study, a preliminary test should be done to determine if the electronic tongue instrument is able to differentiate simple substances with diverse chemical and taste properties. [9] [17] [18]. In order to evaluate the discrimination capability of the sensor array, the instrument was used with a series of five simple substances whose properties are related to coffee including the specificity of basic flavors. Thus, to initially evaluate the differentiation capacity of liquids from the voltammetric and electronic tongue, measures were made on five solutions representing basic flavors related to coffee; $\mathrm{NaCl}\left(0.1 \mathrm{~mol} \mathrm{~L}^{-1}\right)$, sucrose $\left(0.1 \mathrm{~mol} \mathrm{~L}^{-1}\right)$, citric acid $\left(0.1 \mathrm{~mol} \mathrm{~L}^{-1}\right)$, caffeine $\left(0.1 \mathrm{~mol} \mathrm{~L}^{-1}\right)$ y vainillin $\left(0.1 \mathrm{~mol} \mathrm{~L}^{-1}\right)$. On the experiments, seven replicas of each measure were made to evaluate the capacity of reproduction of signals given by the sensor array. On the different registered signals, it was observed that each sensor presents a particular response ('fingerprint') to a type of substance with a determined taste property, producing a sign with informationon the analyzed substance. It is important to point out that with the sensor array it is possible to get voltammetric signals with well-defined peaks (with information on the analyzed method) from non-electroactive substances, differing from that obtained with the use of traditional voltammetric and metallic sensors utilized by other authors [19] [20].

The response of sensor PPy/DBS to the solutions shows a good example of the crossed selectivity from the sensors. The electrochemical behavior from the polypyrrole redox pair to the solutions is shown on Figure 2. The cyclic voltammetry of the PPy/DBS electrodes in contact with $\mathrm{NaCl}$ (salty), citric acid (sour) and caffeine (bitter) showed two redox couples (Figures 2(a)-(c)). The figure shows that the cathodic sweeps were dominated by one sharp reduction peak (at $-0.63 \mathrm{~V},-0.58 \mathrm{~V}$ and $-0.48 \mathrm{~V}$ for $\mathrm{NaCl}$, citric acid and caffeine, respectively) and one smaller, broader peak at ca. $0.09 \mathrm{~V},-0.08 \mathrm{~V}$ and $0.20 \mathrm{~V}$ for $\mathrm{NaCl}$, citric acid and caffeine, respectively. Two redox processes were also observed in the anodic branch (at $-0.55 \mathrm{~V}$ and $0.08 \mathrm{~V}$ for $\mathrm{NaCl},-0.63 \mathrm{~V}$ and -0.05 for citric acid and $-0.71 \mathrm{~V}$ and $-0.07 \mathrm{~V}$ for caffeine). The CV from PPy/DBS in vanillin and sucrose (tow sweet compounds) showed two redox processes. The anodic peaks appear at -0.75 and $-0.25 \mathrm{~V}$ for vanillin and -0.69 and -0.15 for sucrose. In the corresponding reverse scan, the cathodic wave of the first redox couple was not clearly observed, whereas that of the second redox process appeared at $-0.57 \mathrm{~V}$ and $-0.45 \mathrm{~V}$ for vanillin and sucrose respectively. According to the literature, these peaks can be assigned respectively to the formation of the polaron and bipolaron [21].

During the first redox process occurring at lower potentials (peak I), the doping anions remain immobilized inside the film, whereas cations are exchanged with the surrounding solution to maintain the electroneutrality. In the second process (peak II), occurring at higher voltages, the PPy film gets highly oxidized and anions from the electrolyte are inserted into the film to maintain the charge balance [22] [23].

The possible mechanisms that generate the responses of the sensors can be associated with the electrochemi- 


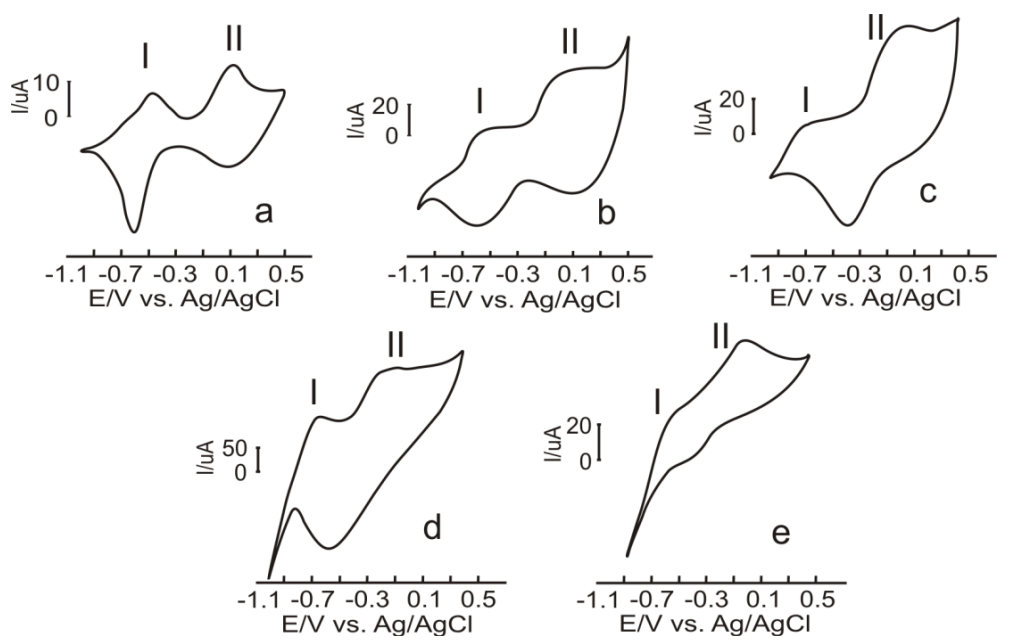

Figure 2. PPy/DBS sensor response against: (a) $\mathrm{NaCl}$, (b) citric acid, (c) caffeine, (d) vanillin y (e) sucrose.

cally controlled ion-exchange process. The exact nature of the ion-exchange process is not clearly deciphered, but it has been established that during the first redox process occurring at lower potentials (peak I), the doping anions remain immobilized inside the film, whereas cations are exchanged with the surrounding solution to maintain the electroneutrality. In the second process (peak II), occurring at higher voltages, the PPy film gets highly oxidized and anions from the electrolyte solution are inserted into the film to maintain the charge balance [24]. The chemical basis of the electrochemical responses can be explained as follows:

On the first reduction/oxidation process for reasons of electroneutrality, the PPy film behaves as a cation exchanger: The reduction/oxidation process leading to the second reduction/oxidation process can be formulated as follows:

In this case, the electroneutrality makes the film behave as an anion exchanger.

The PPy symbolizes a segment of the polypyrrole chain, the subscript indicates that the species is in the solution phase and is in the PPy matrix.

The above results indicate that the shape and position of the peaks depend on the nature of the doping anion. Therefore, a rich variety of sensor responses was achieved by changing the doping agent entrapped inside the polypyrrole films. On the other hand, the ability to incorporate and expel ionic species during the redox transformations for preserving the macroscopic electroneutrality of the electrode can be used to produce analytically useful signals.

In order to study the lifetime of the electrodes, they were recurrently exposed (twenty exposures per day in seven successive days) to an electrolytic solution of $0.1 \mathrm{~mol} \mathrm{~L}^{-1} \mathrm{NaCl}$. Then, the coefficients of variation (\%CV) of the peak heights were calculated. The electrodes showed good stabilities up to the fifth day of exposition, with $\% \mathrm{CV}$ values below $15 \%$. On the seventh day, the \%CV increased drastically ( $30 \%$ in all cases).

The voltammograms on the different solutions show a double process of oxidation/reduction typical of the polymer but in each case the sign is completely different and the potential varies from one solution to the next. The other sensors also showed crossed selectivity, that is, they showed a particular behavior to each substance. Besides, each sensor responds differently to a unique type of substance. Thereby, it is evident that each sensor shows a characteristic response to each solution. These changes are mainly due to variation on forms, positions of redox peaks and relative intensities. This way, the matrix data built from the intensity values of the signals given by the sensor array allowed to make the work of differentiating the substances through an NNPC analysis.

In order to evaluate the discrimination capabilities of the voltammetric sensors, NNPC analysis was conducted by combining the information obtained from the PPy electrodes system. Then, the array of electrodes was immersed in the samples and seven replicates of each sample were registered. Because the voltammograms comprise a large amount of information with a large number of current-potential pairs and since a CV is a bi-valuated curve, the anodic scan was selected to build the matrix used for the pattern recognition techniques.

Figure 3 shows results obtained from the NNPC analysis. This representation of the primary three principal components shows a clear differentiation of the five studied substances (with different taste properties). The three 
principal components represented in the figure present a variance (information) of $87.5 \%$, as expected, the first component (C.P.1) has the maximum information (54.0\%); the second principal component (C.P. 2) contains $20.3 \%$ and the third principal component (C.P.3) only $12.8 \%$.

Having tested the differentiation capacity of the electronic tongue, the instrument was used to analyze coffee samples. Samples were commercially acquired and represent five out of the most popular brands in Colombia. Each sample was analyzed with the sensor array and seven replicas were made for measuring. On the sensors' response it could be observed that each sensor showed a particular response when exposed to each coffee sample. The response variety of the sensors, which reflects the high crossed selectivity of the array, is represented on Figure 4. This response variety is due to interactions that take place among the different sensors and the sample compounds.

Responses from each sensor vary from one coffee brand to the next. As for the case of simple substances with different analyzed taste properties in the preliminary assays, these differences are detected mainly by changes in the peak's position and the variations in its relative intensities. This way, the data given by the electrochemical signals from the sensor array can be utilized to differentiate and classify coffee samples with no need to analyze its particular components.

From the registered signals, a data matrix was built which allowed classifying the coffee samples through an NNPC analysis. Figure 5 shows the resultant graph from the NNPC analysis done from the obtained data. The three primary and principal components summarize $87.0 \%$ of information; the first component collects a $53.9 \%$, the second one a $23.6 \%$ and the third $9.7 \%$. As observed, each of the clusters resulting from the analyzed sample are perfectly differentiated, consequently the sensor array allows to clearly classify the analyzed coffee samples. These results demonstrate that the developed electronic tongue instrument with polymeric sensors can be utilized to perform experiments on classifying coffee samples.

\section{Conclusion}

An electronic tongue elaborated from a polymeric sensors array was used to classifying coffee samples. The electrochemical responses to the analyzed samples were collected through an electronic instrument of multichannel measures (multi-potentiostat) which was able to differentiate a group of substances with different taste

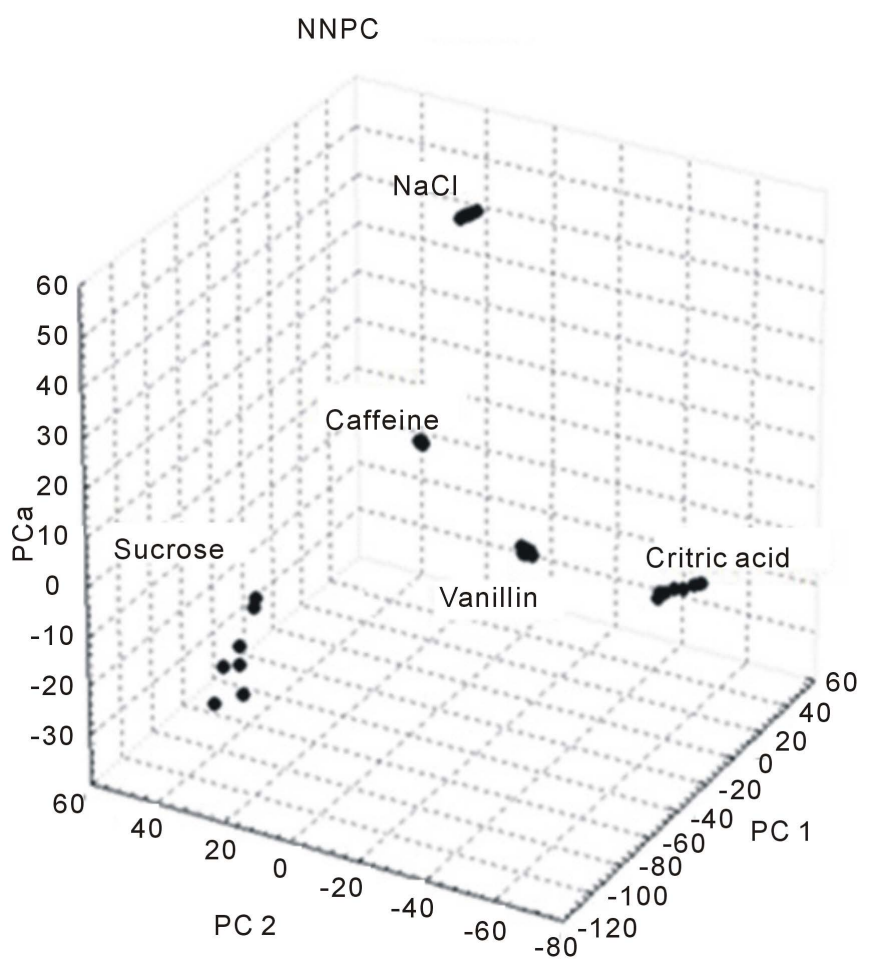

Figure 3. NNPC analysis based on signals matrix obtained from substances with basic taste properties. 


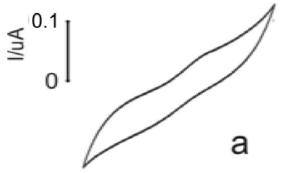

a

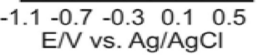

$\unlhd_{0}^{0.1} \mathrm{I}$

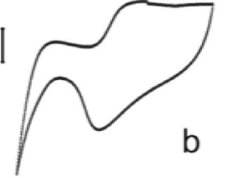

$-1.1-0.7-0.30 .10 .5$
$\mathrm{E} / \mathrm{N}$ vs. Ag/AgCl

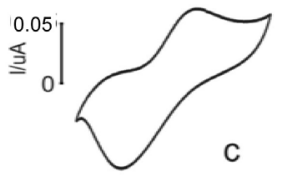

$-1.1-0.7-0.30 .10 .5$ $\mathrm{E} / \mathrm{V}$ vs. $\mathrm{Ag} / \mathrm{AgCl}$
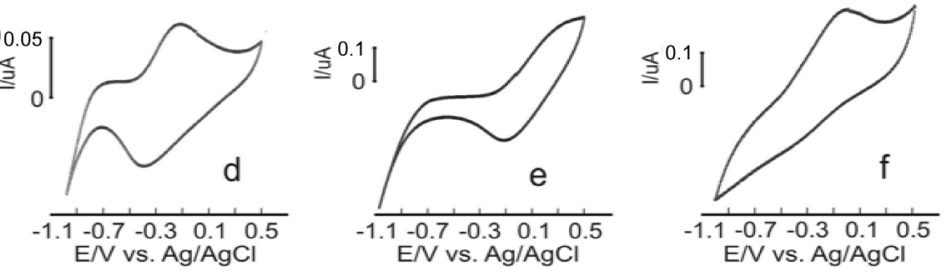

Figure 4. Electronic tongue registered signals against Córdoba coffee: (a) PPy/ AQDS, (b) PPy/DBS, (c) PPy/FCN, (d) PPy/HCF, e) PPy/PWA y (f) PPy/PTS.

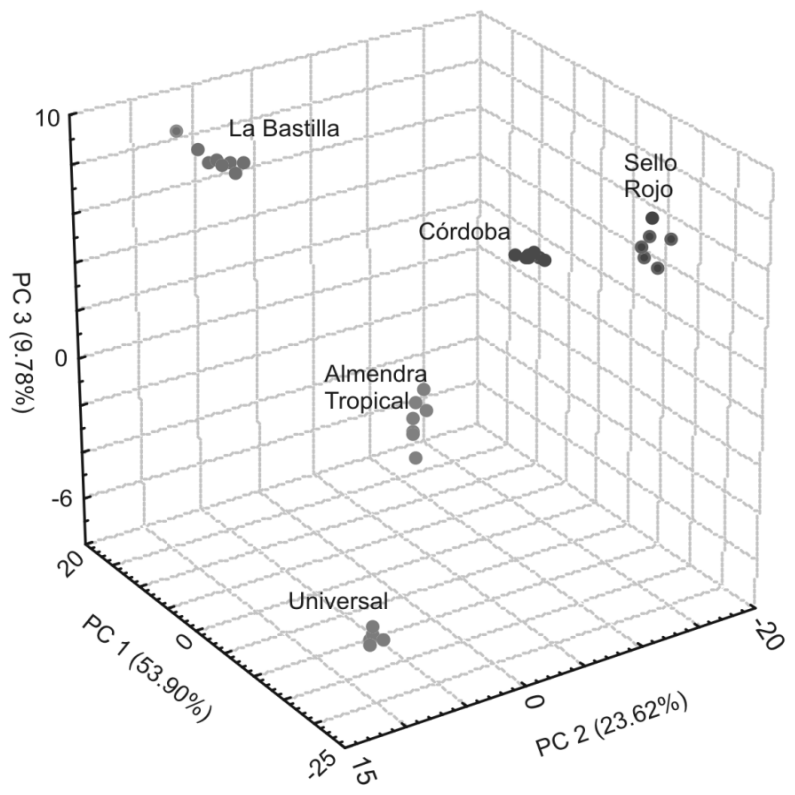

Figure 5. NNPC Analysis based on the electronic tongue response against commercial coffee samples.

properties. The classification of the substances was made through an NNPC analysis and the resultant graphic shows an excellent separation of clusters representing each studied sample. In addition, when this device was used with coffee samples, it showed signals that were converted to a samples' fingerprint since each sensor provides a particular curve that can be used to differentiate the samples using NNPC. This opens the possibility of using these devices as the sensing units of an electronic tongue in the analysis of other beverages and sample types (water, drugs, beer, etc.).

\section{Acknowledgements}

Financial support of Departamento Administrativo de Ciencia, Tecnología e Innovación Colciencias-SENA and Universidad Pontificia Bolivariana—Montería (proyecto RC-675-2008, Código: 1425-452-21195).

\section{References}

[1] Flament, I. (2002) Coffee Flavor Chemistry. John Wiley \& Sons Ltd, Chichester. 
[2] Smith, D.V. and Margolskee, R.F. (2001) Making Sense of Taste. Scientific American, 284, 32-39. http://dx.doi.org/10.1038/scientificamerican0301-32

[3] Norgren, R. (1990) The Gustatory System, The Human Nervous System. George Paxinos, Academic Press.

[4] Arrieta, A.A., Rodríguez Méndez, M.L., De Saja, J.A. and Blanco, C.A. (2010) Prediction of Bitterness and Alcoholic Strength in Beer Using an Electronic Tongue. Food Chemistry, 123, 642-646. http://dx.doi.org/10.1016/j.foodchem.2010.05.006

[5] Arrieta, A.A., Rodríguez Méndez, M.L. and De Saja, J.A. (2010) Aplicación de una Lengua Electrónica Voltamétrica para la Clasificación de vinos y Estudio de Correlación con la Caracterización Química y Sensorial. Quimica Nova, 33, 787. http://dx.doi.org/10.1590/S0100-40422010000400004

[6] Mimendia, A., Legin, A., Merkoçi, A. and Del Valle, M. (2010) Use of Sequential Injection Analysis to Construct a Potentiometric Electronictongue: Application to the Multidetermination of Heavy Metals. Sensors and Actuators B: Chemical, 146, 420-426. http://dx.doi.org/10.1016/j.snb.2009.11.027

[7] Toko, K., Hayashi, K., Yamanaka, M. and Yamafuji, K. (1990) Technical Digest of 9th Sensor Symposium. 193.

[8] Parra, V., Arrieta, A.A., Fernández Escudero, J.A., Rodríguez Méndez, M.L. and De Saja, J.A. (2006) Electronic Tongue Based on Chemically Modified Electrodes and Voltammetry for the Detection of Adulterations in Wines. Sensors and Actuators B: Chemical, 118, 448-453. http://dx.doi.org/10.1016/j.snb.2006.04.043

[9] Wei, Z. and Wang, J. (2011) Detection of Antibiotic Residues in Bovine Milk by a Voltammetric Electronictongue System. Analytica Chimica Acta, 694, 46-56. http://dx.doi.org/10.1016/j.aca.2011.02.053

[10] Chen, Q., Zhao, J. and Vittayapadung, S. (2008) Identification of the Green Tea Grade Level Using Electronic Tongueand Pattern Recognition. Food Research International, 41, 500-504. http://dx.doi.org/10.1016/j.foodres.2008.03.005

[11] Woertz, K., Tissen, C., Kleinebudde, P. and Breitkreutz, J. (2011) A Comparative Study on Two Electronic Tongues for Pharmaceutical Formulation Development. Journal of Pharmaceutical and Biomedical Analysis, 55, 272-281. http://dx.doi.org/10.1016/j.jpba.2011.02.002

[12] Sun, H., Mo, Z.H., Choy, J.T.S., Zhu, D.R. and Fung, Y.S. (2008) Piezoelectric Quartz Crystal Sensor for Sensing Taste-Causing Compounds in Food. Sensors and Actuators B: Chemical, 131, 148-158. http://dx.doi.org/10.1016/j.snb.2007.12.014

[13] Leonte, I.I., Sehra, G., Cole, M., Hesketh, P. and Gardner, J.W. (2006) Taste Sensors Utilizing High-Frequency SHSAW Devices. Sensors and Actuators B: Chemical, 118, 349.

[14] Veríssimo, M.I.S., Oliveira, J.A.B.P. and Gomes, M.T.S.R. (2010) Contribution of Compressional Waves to the Identification and Quantification of a Water Contaminant. Sensors and Actuators B: Chemical, 151, 21-25. http://dx.doi.org/10.1016/j.snb.2010.09.065

[15] Bogomolov, A. (2011) Multivariate Process Trajectories: Capture, Resolution and Analysis. Chemometrics and Intelligent Laboratory Systems, 108, 49-63. http://dx.doi.org/10.1016/j.chemolab.2011.02.005

[16] Oliveri, P., Casolino, M.C. and Forina, M. (2010) Chapter 2-Chemometric Brains for Artificial Tongues. Advances in Food and Nutrition Research, 61, 57-117. http://dx.doi.org/10.1016/B978-0-12-374468-5.00002-7

[17] Pioggia, G., Di Francesco, F., Marchetti, A., Ferro, M., Leardi, R. and Ahluwalia, A. (2007) A Composite Sensor Array Impedentiometric Electronic Tongue Part II. Discrimination of Basic Tastes. Biosensors and Bioelectronics, 22, 2624-2628. http://dx.doi.org/10.1016/j.bios.2006.10.025

[18] Arrieta, A., Rodriguez-Mendez, M.L. and De Saja, J.A. (2003) Langmuir-Blodgett Film and Carbon Paste Electrodes Based on Phthalocyanines as Sensing Units for Taste. Sensors and Actuators B: Chemical, 95, 357-365. http://dx.doi.org/10.1016/S0925-4005(03)00438-6

[19] Tian, S., Deng, S. and Chen, Z. (2007) Multifrequency Large Amplitude Pulse Voltammetry: A Novel Electrochemical Method for Electronic Tongue. Sensors and Actuators B: Chemical, 123, 1049-1056. http://dx.doi.org/10.1016/j.snb.2006.11.011

[20] Söderström, C., Borén, H., Winquist, F. and Krantz-Rülcker, C. (2003) Use of an Electronic Tongue to Analyze Mold Growth in Liquid Media. International Journal of Food Microbiology, 83, 253-261. http://dx.doi.org/10.1016/S0168-1605(02)00375-6

[21] Arrieta, A., Apetrei, C., Rodríguez Méndez, M.L. and De Saja, J.A. (2004) Voltammetric Sensor Array Based on Conducting Polymer-Modified Electrodes for the Discrimination of Liquids. Electrochimica Acta, 49, 4543-4551. http://dx.doi.org/10.1016/j.electacta.2004.05.010

[22] Martini, M. and De Paoli, M. (2002) Effect of the Electrolyte Cations and Anions on the Photocurrent of Dodecylsulphate Doped Polypyrrole Films. Solar Energy Materials and Solar Cells, 73, 235-247. http://dx.doi.org/10.1016/S0927-0248(01)00133-7 
[23] Syritski, V., Opik, A. and Forsen, O. (2003) Ion Transport Investigations of Polypyrroles Doped with Differentanions by EQCM and CER Techniques. Electrochimica Acta, 48, 1409-1417. http://dx.doi.org/10.1016/S0013-4686(03)00018-5

[24] Zhao, C. and Jiang, Z. (2004) Polymerization and Redox Behavior of Polypyrrole (PPy) Films by in Situ EQCM and PT Techniques. Applied Surface Science, 229, 372-376. http://dx.doi.org/10.1016/j.apsusc.2004.02.014 